\title{
Edible Bird's Nest, an Asian Health Food Supplement, Possesses Skin Lightening Activities: Identification of N-Acetylneuraminic Acid as Active Ingredient
}

\author{
Gallant Kar Lun Chan, Zack Chun Fai Wong, Kelly Yin Ching Lam, Lily Kwan Wai Cheng, \\ Laura Minglu Zhang, Huangquan Lin, Tina Tingxia Dong, Karl Wah Keung Tsim* \\ Division of Life Science and Center for Chinese Medicine R\&D, The Hong Kong University of Science and \\ Technology, Kowloon, Hong Kong, China \\ Email: "botsim@ust.hk
}

Received 29 September 2015; accepted 23 November 2015; published 27 November 2015

Copyright (C) 2015 by authors and Scientific Research Publishing Inc.

This work is licensed under the Creative Commons Attribution International License (CC BY).

http://creativecommons.org/licenses/by/4.0/

(c) (i) Open Access

\begin{abstract}
Edible bird's nest (EBN; Yan Wo), or cubilose, is originated from the salivary secretion of Aerodramus fuciphagus. In Asia, EBN is famous for its unproven skin lightening function. Here, we aim to reveal the active ingredients of EBN responsible for skin lightening function. Three major fractions were isolated from EBN water extract by chromatography using LC-MS/MS, bioactivities of these isolated fractions were analyzed by assays of tyrosinase, melanocytes and 3D human skin model, from which, $\mathrm{N}$-acetylneuraminic acid (NANA), the second isolated fraction showed an inhibition effect on tyrosinase activity in a dose-dependent manner. The $\mathrm{IC}_{50}$ of tyrosinase originated from mushroom and human was $16.93 \mathrm{mM}$ and $0.10 \mathrm{mM}$ respectively. Furthermore, only EBN with higher content of NANA (e.g. White and Red EBN), but not EBN with less NANA (e.g. Grass EBN), showed promising skin lightening function. This is the first report to reveal NANA being an active ingredient of EBN on skin lightening function.
\end{abstract}

\section{Keywords}

Cubilose, N-Acetylneuraminic Acid, Chemical Analysis, Skin Lightening, Human Skin Model

\footnotetext{
${ }^{*}$ Corresponding author.
}

How to cite this paper: Chan, G.K.L., et al. (2015) Edible Bird's Nest, an Asian Health Food Supplement, Possesses Skin Lightening Activities: Identification of N-Acetylneuraminic Acid as Active Ingredient. Journal of Cosmetics, Dermatological Sciences and Applications, 5, 262-274. http://dx.doi.org/10.4236/jcdsa.2015.54032 


\section{Introduction}

Edible bird's nest (EBN; Yan Wo), or named cubilose, is made of the salivary secretion of specific swiftlets (e.g. Aerodramus fuciphagus). EBN is an ingredient of an ancient Chinese delicacy-the EBN soup, which has been consumed for several hundred years in Asia according to the historical record. Until now, EBN is still a popular luxurious food supplement for women in the oriental population. However, the biological functions of EBN are still unclear.

There are two major problems in studying the biological functions of EBN. Firstly, the progress of mechanistic study is largely hindered by our insufficient knowledge on the bioactive ingredients of EBN. EBN consists of $40 \%-60 \%$ of protein, $9 \%$ of sialic acid and trace amount of fat and minerals [1]. However, those chemical parameters are too superficial to unveil the biological functions of EBN. Secondly, there is a large variety of EBN on the market, and there is no standardized EBN for biological determinations (e.g. skincare functions). Even worse, most of the current authentication methods of EBN, including microscopic [2], proteomics [3] and genomics [4] approaches, all fail to differentiate different grades of EBN [5].

The free form of $\underline{N}$-acetylneuraminic acid (NANA) could serve as a quantitative marker to grade different EBNs [6] [7]. The abundant of which increases in line with higher prices of EBN. Furthermore, NANA is known to have biological activities. NANA was proposed to be a major component for anti-influenza function of EBN [8] [9]. Moreover, NANA has proliferation effect on Caco-2 cells [10]. However, the biological functions of NANA on skin functions are unknown. Furthermore, skincare-related bioactive compounds other than protein and NANA might also be found in EBN. Thus, a full scanning of total ion chromatography was performed by LC-MS/MS, and the performances on skincare functions by different fractions were analyzed.

\section{Materials and Methods}

\subsection{Material}

NANA was purchased from Sigma-Aldrich (St. Louis, MO) as standard solution. Volume were measured accurately from the stock, diluted with fresh Milli-Q to produce a series of solution standards $(1,2,5,10,15,20 \mu \mathrm{M})$. Tyrosinase from mushroom ( $\geq 1000 \mathrm{U} / \mathrm{mg}$ ) and recombinant human tyrosinase (Activity $\geq 95 \%$ ) were purchased from Sigma-Aldrich. L-3, 4-dihydroxyphenyl-alanine (L-DOPA), vitamin C and tert- Butylhydroquinone (tBHQ) and alpha melanocyte stimulating hormone $(\alpha-\mathrm{MSH})$ were purchased from Sigma-Aldrich. All EBN samples and its adulterants were purchased in the market of Hong Kong.

\subsection{Sample Preparation}

For LC-MS/MS analysis, EBN samples were ground into powder (approximately 1 - $3 \mathrm{~mm}$ ) and mixed thoroughly. Ten mg of each was weighed and extracted by $1 \mathrm{~mL}$ of fresh Milli-Q water under sonication for 10 min. Followed by centrifugation at 14,000 rpm for $5 \mathrm{~min}$, the supernatants were filtered, and the filtrates were collected for LC-MS/MS analysis. For bioassay, one g of EBN sample, or its adulterants, was individually soaked in 100 folds of water for overnight at room temperature. The mixture was then stewed at $80^{\circ} \mathrm{C}$ for $6-9$ hours until completely molten and then left to cool down to room temperature. This method of preparation is a common cooking method for the consumers, as well as a high protein extraction efficiency [11]. The extracts were kept at $4^{\circ} \mathrm{C}$ until further usage.

\subsection{HPLC-MS/MS System}

The liquid chromatograph was equipped with an Agilent 6410 Triple Quad MS/MS and a $(2.1 \times 100 \mathrm{~mm})$ Eclipse XDB-C18 column (3.5 $\mu \mathrm{m}$ particle size). The injection volume was $2 \mu \mathrm{L}$. A 5-min linear gradient at flow rates of $0.4 \mathrm{~mL} / \mathrm{min}$ between solvent A (Milli-Q water, $0.1 \%$ formic acid) and solvent B (Acetonitrile, $0.1 \%$ formic acid) was used. After reaching $80 \% \mathrm{~B}$, the system returned to $100 \% \mathrm{~A}$ in $0.5 \mathrm{~min}$. For column equilibration, a total cycle time of 10 min was needed. The MS was operated in negative electron spray ionization mode. A capillary voltage of $3.5 \mathrm{kV}$ and a cone voltage of $10 \mathrm{~V}$ were applied. Source temperature was $100^{\circ} \mathrm{C}$, and desolvation temperature was $325^{\circ} \mathrm{C}$. Ultra-high purity nitrogen was used for cone gas $(3.0 \mathrm{~L} / \mathrm{min})$, desolvation gas $(10.0 \mathrm{~L} / \mathrm{min})$ and nebulising gas (35 psi). For collision induced dissociation (CID) a collision energy of $5 \mathrm{eV}$ was used. Spectra from m/z 50 to m/z 1000 were recorded. 


\subsection{Quantification of NANA and Citric Acid}

In calibration of NANA in EBN, a 5-point calibration curve having concentrations of 1, 2, 5, 10 and $20 \mu \mathrm{M}$ was made. All calibrators were prepared in fresh Milli-Q water. Triplicate results were taken for each sample. Retention time of NANA was $0.65-0.69$ min. A negatively single charged ion $[\mathrm{M}-\mathrm{H}]^{-}$of NANA (m/z 307.9) was selected as precursor ion for CID. The precursor ion was dissociated into two major product ions $(\mathrm{m} / \mathrm{z} 87.0$ and 170.0), and the product ion $\mathrm{m} / \mathrm{z} 87.0$ was the most abundant from NANA. Multiple reaction monitoring (MRM) was applied, the transitions $\mathrm{m} / \mathrm{z} 307.9 \rightarrow 87.0$ and $\mathrm{m} / \mathrm{z} 307.9 \rightarrow 170.0$ were chosen as the qualifiers, whilst the transitions $\mathrm{m} / \mathrm{z} 307.9 \rightarrow 87.0$ was measured for quantification. The determination of citric acid was similar to that of NANA. The retention time of citric acid was at $1.50 \mathrm{~min}$. The solution having known spiked amount of citric acid was defined as the quality control (QC) solution. Negatively single charged ion $[\mathrm{M}-\mathrm{H}]^{+}$of citric acid $(\mathrm{m} / \mathrm{z}$ 191.0) was selected as precursor ion for CID. The precursor ion was dissociated into two major product ions (m/z 111.0 and 86.9), and the product ion $\mathrm{m} / \mathrm{z} 111.0$ was the most abundant from NANA. Multiple reaction monitoring was applied, the transitions $\mathrm{m} / \mathrm{z} 191.0 \rightarrow 111.0$ and $\mathrm{m} / \mathrm{z} 191.0 \rightarrow 86.9$ were chosen as the qualifiers, whilst the transitions $\mathrm{m} / \mathrm{z} 191.0 \rightarrow 111.0$ was measured for quantification. The ethyl $\beta$-D-glucuronide $(\mathrm{m} / \mathrm{z}$ 221.1) was used as the internal standard in the calibrators and QC samples, and the concentration was kept consistent at $100 \mathrm{ng} / \mathrm{mL}$. The transitions $\mathrm{m} / \mathrm{z} 221.1 \rightarrow 84.9$ and $\mathrm{m} / \mathrm{z} 221.1 \rightarrow 75.0$ were chosen as the qualifiers, whilst the transitions $\mathrm{m} / \mathrm{z} 221.1 \rightarrow 84.9$ was quantified as reference.

\subsection{Tyrosinase Assay}

The enzyme activities of mushroom and human tyrosinase were monitored by dopachrome formation at $492 \mathrm{~nm}$ through the oxidation of substrate (L-DOPA). The reaction medium $(200 \mu \mathrm{L})$ contained $0.5 \mathrm{mM}$ L-DOPA in 50 $\mathrm{mM}$ sodium phosphate buffer ( $\mathrm{pH}$ 6.8) and ( $\mathrm{pH}$ 7.4). Five mM of vitamin $\mathrm{C}$ was used as an inhibitor control. The final concentration of mushroom tyrosinase was $0.2 \mathrm{mg} / \mathrm{mL}$. In this method, $0.1 \mathrm{~mL}$ of different concentrations of effectors, including EBN extracts, adulterants of EBN and NANA, were added to the reaction medium. The reaction mixtures were loaded on a 96-well plate, and the formation of dopachrome was measured in optical density at $492 \mathrm{~nm}$ after 20 min of incubation under dark at room temperature. Absorption was recorded using micro-plate spectrophotometer.

\subsection{Melanogenesis Assay on Human and Mouse Melanocytes}

B16 cells (CRL-6323тм; American Type Culture Collection (ATCC), Manassas, VA) and A375 cells (CRL$1619^{\mathrm{TM}}$, ATCC) were purchased from ATCC and cultured according to the recommendations provided. B16 and A375 cells were cultured in Dulbecco's Modified Eagle's Medium (DMEM) supplemented with 10\% fetal bovine albumin and Penicillin-Streptomycin $(100 \mathrm{U} / \mathrm{mL})$. Cells were sub-cultured every alternate day.

For melanogenesis assay, two hundred thousands of B16 cells and A375 cells were seeded onto each well of 6-well culture plates and incubated for 2 days, or 4 days, with or without the extracts of EBN, respectively. Five $\mathrm{mM}$ of vitamin C, $50 \mu \mathrm{M}$ of tBHQ and $2 \%$ kojic acid served as positive controls. For A375 cells, $\alpha$-MSH was added on day 2 as to stimulate the formation of melanin. To extract melanin from cells, the samples were dissolved in $100 \mathrm{~mL}$ of $1 \mathrm{M} \mathrm{NaOH}$ after washing twice with PBS. Samples were incubated at $60^{\circ} \mathrm{C}$ for 1 hour and mixed to solubilize the melanin. The optical density of mixed solution was detected at $405 \mathrm{~nm}$, and the absorbance was converted to melanin concentration by a standard curve.

\subsection{D Human Skin Model Assay}

Twenty-four 3D human skin model constructed with keratinocytes and melanocytes in a ratio of originated from Asian skin tissue were purchased from MatTek Corporation (Lot \# 21822). The model was specific for melanogenesis study (MelanoDerm ${ }^{\mathrm{TM}}$ Part \# MEL-300-A). All procedures for macroscopic and microscopic analysis followed the instructions provided by the manufacturer. In brief, the testing samples (20 mM NANA and 1 $\mathrm{mg} / \mathrm{mL}$ EBN extract) and positive control (2\% of kojic acid) were applied either to the inner chambers of trans-well (keratinocyte side) or culture medium directly (melanocyte side). Culture medium having same concentration of tested chemicals was replaced every 2 - 3 days. The inner chamber was rinsed twice with phosphate buffer provided by the manufacturer and replaced with fresh testing samples and positive control. Macroscopic and microscopic images were captured on day 18. Macroscopic images were captured by top view 
microscope (keratinocyte side), while microscopic images were captured by inverted microscope under 100× magnification (melanocyte side).

\subsection{Protein Assay and Statistical Tests}

The concentration of protein was determined following the instructions of Bradford's method with a kit from Bio-Rad Laboratories. The analysis was done on a 96-well microtiter plate. In brief, one part dye reagent concentrate was diluted with 4 parts of double distilled water before use. Six dilutions of BSA standard (0.05 - 0.5 $\mathrm{mg} / \mathrm{mL}$ ) were used for the test: Ten $\mu \mathrm{L}$ of each standard, or a sample solution, were added with $0.2 \mathrm{~mL}$ of diluted dye reagent into separate wells and mixed well. After 10 min incubation at room temperature, absorbance at $595 \mathrm{~nm}$ was taken. The concentration of protein was determined from the standard curve. Statistical tests were done by using student $t$ test provided in GraphPad Prism 5.0. Statistically significant changes were classed as [*] where $\mathrm{p}<0.05$, $[* *]$ where $\mathrm{p}<0.01$ and $[* * *]$ where $\mathrm{p}<0.001$.

\section{Results and Discussion}

\subsection{Fractionation of EBN by LC-MS/MS}

EBNs are graded and priced according to their color, i.e. Red $>$ White $>$ Grass. These EBNs were collected from local market, and which were subjected to LC-MS/MS analysis. Three major fractions (named peak 1, 2 and 3) from EBN water extracts were notified and appeared at different retention time after the separation using the full scanning mode of LC-MS/MS chromatography (Figure 1(a)). Interestingly, the full scans of total ion chromatogram of different grades of EBN showed differentiable patterns. The full scan of Grass EBN showed a strong peak 1 and a barely recognizable peak 2. The full scan of White EBN showed both notable peaks 1 and 2 . While the full scan of Red EBN showed all three distinguishable 3 peaks. After the analysis with molecular weight using MRM mode quantitation and the chemical standards, the identities of peak 2 and peak 3 were confirmed as NANA and citric acid, respectively (Figure 1(b)). Peak 1 consisted of compounds with different sizes, and the pattern of mass spectrum was similar to those spectrums of collagen-like proteins (data not shown). Having the identities of NANA and citric acid, the amounts of them in different grades of EBN were quantified by the established MS system. The contents of protein, free NANA and citric acid for different grades of EBN were summarized (Supplementary Table). The protein contents for different types of EBNs were rather similar; however, the contents of NANA and citric acid were significantly higher in Red EBN and lower in Grass EBN. Citric acid was not detected in Grass EBN.

For safety reason, EBN needs to be proper processed before the consumption by human [11]. As NANA and citric acid are the most abundant soluble chemicals and therefore considered to be the targeted inhibitors. Here, we aimed to reveal the amount of NANA and citric acid in EBN after standard processing procedures. Different sample preparation procedures were applied onto EBN preparation to mimic the standard cooking processes. The content of free NANA increased during the cooking processes, and an increase of $\sim 4$ folds after stewing in different types of EBN (Supplementary Figure 1(a)). The increase could be an outcome of the release of conjugated NANA as the free form after extensive cooking. In contrast, the content of citric acid dropped by $90 \%$ after the processing (Supplementary Figure 1(b)), suggesting the free salt form of citric acid within EBN. Thus, the cooking EBN could result in different amounts of NANA and citric acid.

\subsection{Skin Lightening Effect of EBN}

Tyrosinase inhibition assay is a commonly used method in screening skin lightening agents [12]-[15]. The activity of tyrosinase (mushroom) was calculated on the formation of dopachrome from L-DOPA against time: the concentration of dopachrome was obtained by absorbance at $492 \mathrm{~nm}$ (Figure 2(a)). The inhibition of mushroom tyrosinase was determined in different EBN water extracts. Vitamin $\mathrm{C}$ was used as a positive control. White and Red EBNs inhibited the activity of tyrosinase by about 50\% (Figure 2(b)). In contrast, Grass EBN reduced the tyrosinase activity of only $\sim 10 \%$. No inhibitory functions on tyrosinase for all common EBN adulterants, e.g. agar, fungi and pig skin. In order to search for ingredient corresponding for tyrosinase inhibition, the proteindepleted fraction of EBN water extract was tested. In different types of EBN, the inhibiting activity was enriched in the protein-depleted fraction (Figure 2(c)), which suggested the role of water soluble small chemicals in such function. 
(a)
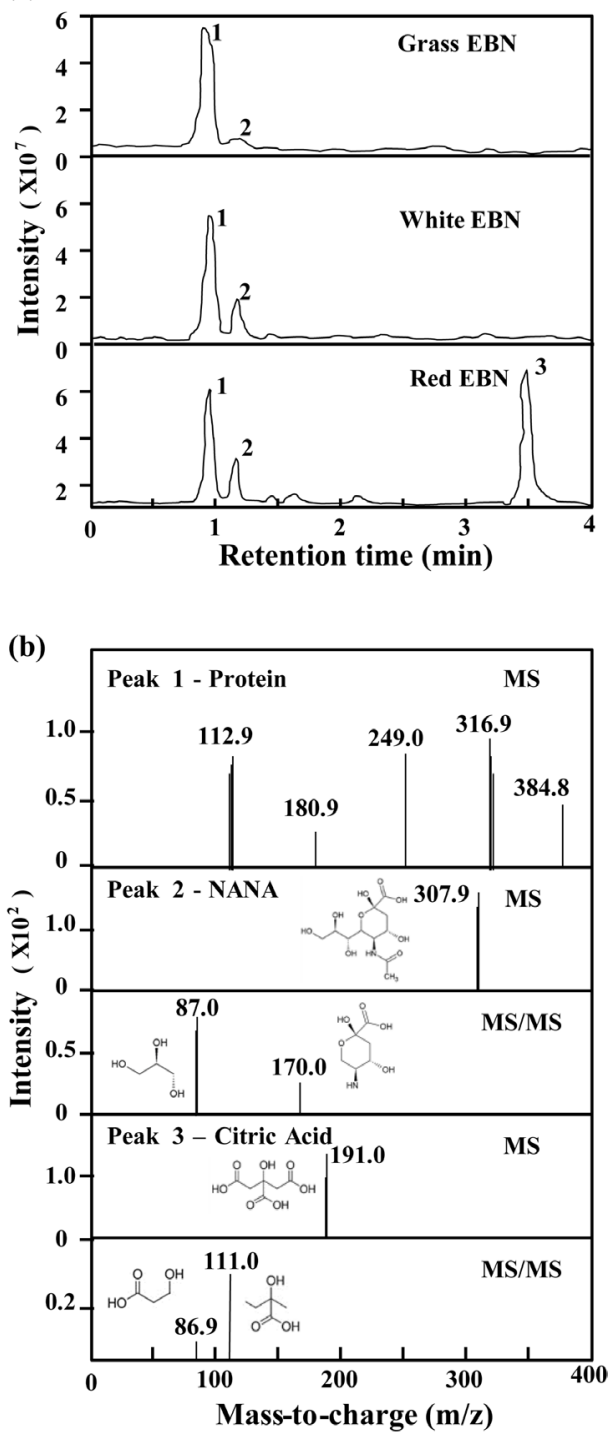

Figure 1. Screening of major ingredients from EBN by LC-MS/MS. (a) Full scan of total ion chromatography of different water extracts of different grades of EBNs. Three outstanding peaks with the highest abundance were recorded along with the retention time. (b) Mass spectrums of the outstanding peaks from the full scan were obtained. From which, peak 1 were identified as protein-like molecules while peak 2 and peak 3 were identified as NANA $(\mathrm{m} / \mathrm{z} 307.9 \rightarrow 87.0$ and $\mathrm{m} / \mathrm{z} 307.9 \rightarrow 170.0)$ and citric acid $(\mathrm{m} / \mathrm{z} 191.0 \rightarrow 111.0$ and $\mathrm{m} / \mathrm{z} 191.0 \rightarrow 86.9)$ respectively. NANA and citric acid standards were applied for the identification. $n=4$.

\subsection{NANA Shows Skin Lightening}

The skin lightening effect by NANA becomes the focus of study here. Having tyrosinase assay, NANA inhibited both mushroom and human tyrosinases in a dose-dependent manner (Figure 3(a)). The $\mathrm{IC}_{50}$ of NANA on mushroom tyrosinase and human tyrosinase was $16.93 \mathrm{mM}$, and $0.10 \mathrm{mM}$, respectively (Figure 3(a)). The original Km for mushroom and human tyrosinase on L-DOPA were $1.491 \mathrm{mM}$ and $0.545 \mathrm{mM}$, respectively; and Vmax were $0.394 \mu \mathrm{M} / \mathrm{min}$ and $0.308 \mu \mathrm{M} / \mathrm{min}$, respectively. Observed from Lineweaver-Burk plot, the values of $\mathrm{Km}$ and Vmax of tyrosinase were altered in the presence of NANA: the changes were in a dose-dependent manner (Figure 3(b)). By adding $10 \mathrm{mM}$ NANA to the mushroom enzyme, the $\mathrm{Km}$ increased from 1.491 to $1.818 \mathrm{mM}$, while the Vmax decreased from 0.394 to $0.287 \mu \mathrm{M} / \mathrm{min}$. In human tyrosinase, the effect of NANA did not 
(a)
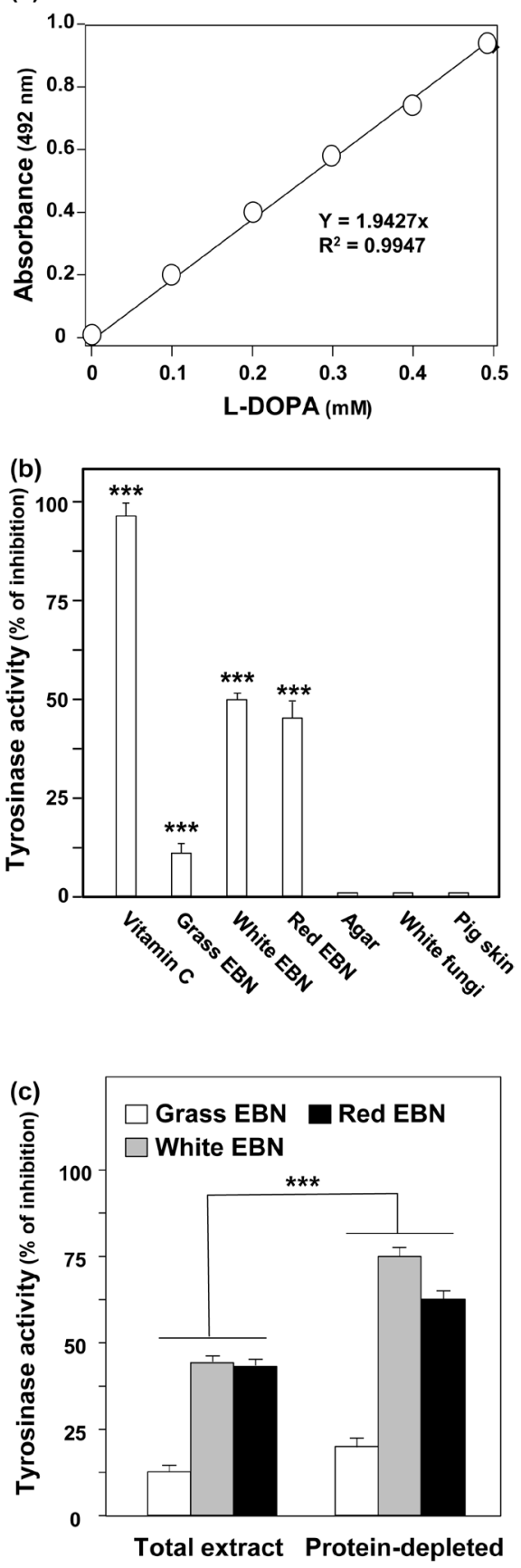

Figure 2. EBNs inhibit the activity of tyrosinase. (a) Different concentrations of L-DOPA were added to testing reagent containing mushroom tyrosinase (5000 $\mathrm{U} / \mathrm{mL}$ ) and incubated for 1 hour. Absorbance readings were taken at $492 \mathrm{~nm}$. (b) The dopachrome formed by oxidation of $0.5 \mathrm{mM}$ of L-DOPA incubated for 20 min with $0.2 \mathrm{mg} / \mathrm{mL}$ mushroom tyrosinase, served as blank control. Vitamin C ( $5 \mathrm{mM})$ served as a positive control. The dopachrome concentration, after treatment of different EBNs or adulterants (all at $10 \mathrm{mg} / \mathrm{mL}$ water extract), was determined. (c) Protein was depleted from the water extracts of different EBNs by precipitation using acetonitrile. The percentage of inhibition on tyrosinase activities before and after protein depletion was recorded. One $\mathrm{mg} / \mathrm{mL}$ extract was added. The percentage of inhibition on tyrosinase activities was presented as Mean $\pm \operatorname{SD}(n=3) .{ }^{* * *} \mathrm{p}<0.001$ versus reference group. 


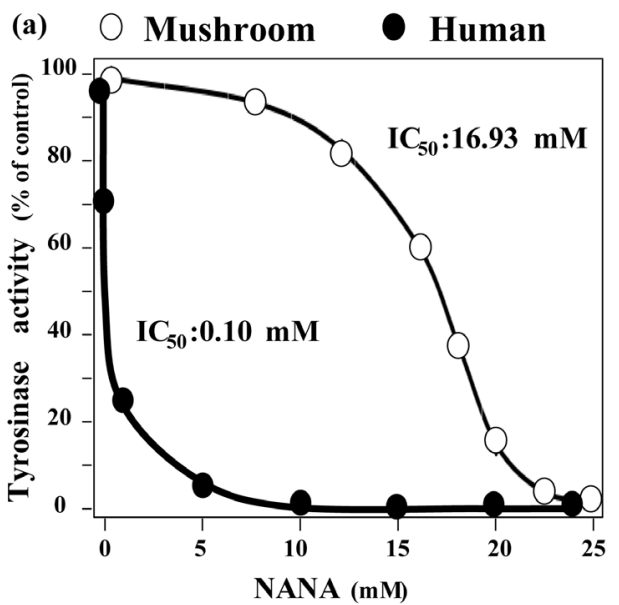

(b)

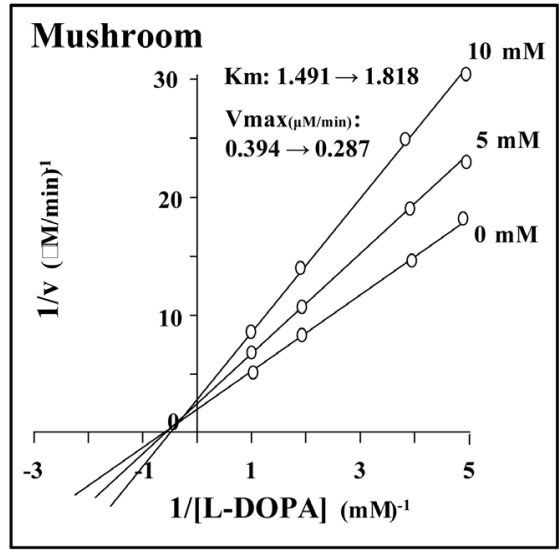

(c)

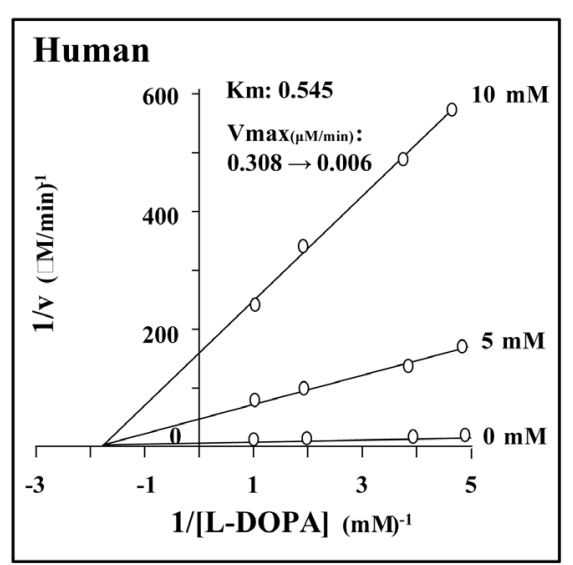

Figure 3. NANA inhibits tyrosinase (a) The reaction rate of mushroom and human tyrosinase was defined as $100 \%$ activity (i.e. $29.75 \mu \mathrm{M} / \mathrm{min}$ and 147.20 $\mathrm{nM} / \mathrm{min}$, respectively) with the substrate of $0.5 \mathrm{mM}$ of L-DOPA. The inhibition effects of NANA from $0 \mathrm{mM}$ to $25 \mathrm{mM}$ on the mushroom and human tyrosinase activity were shown. The reaction velocity was calculated by the concentration of dopachrome, measured by spectrometer at $492 \mathrm{~nm}$ against time in min. Lineweaver-Burk plot of different concentrations of NANA on (b) mushroom tyrosinase and (c) human tyrosinase. Values are Mean \pm SD $(n=3)$. 
change the Km value, while the Vmax decreased from 0.308 to $0.006 \mu \mathrm{M} / \mathrm{min}$ (Figure 3(c)). Referring to the Lineweaver-Burk plot, the inhibition mechanism of NANA on mushroom tyrosinase was classified as mixed type I inhibition, whilst the inhibition mechanism of NANA on human tyrosinase was classified as non-competitive inhibition. Enzymatic parameters of other known tyrosinase inhibitors were compared (Table 1). The enzymatic parameters of inhibition by NANA were comparable to those existing known inhibitors.

In cultured B16 mouse melanoma cells, the color of culture medium was turned into dark brown after 48 hours of culture (Figure 4(a)). This phenomenon was due to formation and release of melanin-rich melanosome from B16 melanoma [16]. For A375 human melanoma, after stimulated by $\alpha$-MSH, culture medium was also turned into dark brown after 48 hours of culture (Figure 4(c)) Vitamin C, tBHQ and 2\% kojic acid, served as positive control, successfully abolished the formation of melanin, and hence the medium color remained unchanged, i.e. inhibition of melanin formation (Figure 4(a) and Figure 4(c)). The melanin concentration extracted from the treated B16 mouse melanoma and A375 human melanoma also agreed with these observations. The melanin concentration of cultured B16 and A375 were significantly lower than the blank control after incubation with NANA and White or Red EBN extract; however, the abolishing effect was not found by the extract of Grass EBN (Figure 4(b) and Figure 4(d)).

In 3D human skin model, treatment of $2 \%$ kojic acid successfully lightened the apparent intensity of skin color (Figure 5(a)) and reduced the density of melanocytes (Figure 5(b)). Similar inhibitory results were obtained by NANA and the extract of White EBN (Figure 5(a), Figure 5(b)). No significant differences in inhibition effects between treatments on the sides of keratinocyte and melanocyte. Thus, kojic acid, NANA and EBN extracts were all suggested to be permeable through the 3D human skin model (Figure 5(a), Figure 5(b)).

\section{Conclusions}

EBN has been consumed for several hundred years in China and other Southeast Asian countries. In the descriptions of ancient Chinese literatures, EBN was often used to treat respiratory disorder. The most famous record about EBN usage, as the form of "bird's nest congee", can be found in "Dream of the Red Chamber", or called that as "The Story of the Stone", a masterpiece of Chinese literature written in $18^{\text {th }}$ century and is generally acknowledged to be the pinnacle of Chinese fiction. Until now, EBN is a popular food supplement for skin lightening. The trend of EBN consumption is growing [17] but stopped by the safety incident of nitrite in 2011 [11]. Although EBN has a long historical consumption record and strong belief in its skincare functions by the general public in Asia is reported, no scientific evidence has shown any relationship between EBN and skin healthiness. Here, we demonstrated the skin lightening functions of different types of EBN. On top of this, we discovered that NANA was the major ingredient of EBN responsible for the skincare functions after systematic fractionation by LC-MS/MS.

From our previous finding, the content of free NANA varied with the grading of EBN [6]. Coincidently, EBN with higher grade showed stronger skin lightening. NANA should be one of the major compounds responsible for the skin lightening function of EBN. However, NANA should not be the only compound within EBN, which is responsible for skin lightening function. The IC $_{50}$ of NANA on human tyrosinase inhibition is $0.10 \mathrm{mM}$ which is coherent to our previous study on over a hundred batches of EBNs. The maximum content of free NANA was around $1000 \mathrm{ppm}$ (i.e. about $3 \mathrm{mM}$ ). Certainly, the inhibition of EBN on tyrosinase activity may also involve the

Table 1. Comparison of NANA and other skin lightening agents.

\begin{tabular}{ccc}
\hline Skin lightening agent & IC $_{\mathbf{5 0}}{ }^{\mathbf{a}}$ & Inhibitory mechanism $^{\mathbf{b}}$ \\
\hline Hydroquinone $^{\mathrm{c}}$ & $0.037 \mathrm{mM}$ & Competitive \\
Arbutin & $24.0 \mathrm{mM}$ & Competitive \\
Gallic acid & $4.50 \mathrm{mM}$ & Unknown \\
Kojic acid & $0.030 \mathrm{mM}$ & Unknown \\
NANA (Sialic acid) & $0.100 \mathrm{mM}$ & Non-competitive \\
\hline
\end{tabular}

${ }^{\mathrm{a}} \mathrm{IC}_{50}$ value was calculated by GraphPad 5.0 after plotting the activity of mushroom tyrosinase against the concentration of different inhibitors, were presented $(n=3)$. ${ }^{\mathrm{b}}$ The inhibitory mechanism of different inhibitors on tyrosinase was deducted by Lineweaver-Burk plot and calculated by GraphPad 5.0. 'Hydroquinone was banned by FDA in 2006 for its potential carcinogenicity. 
(a)
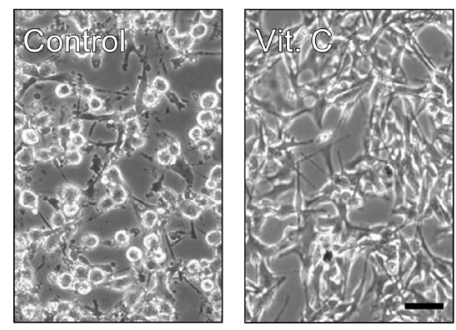

(b)

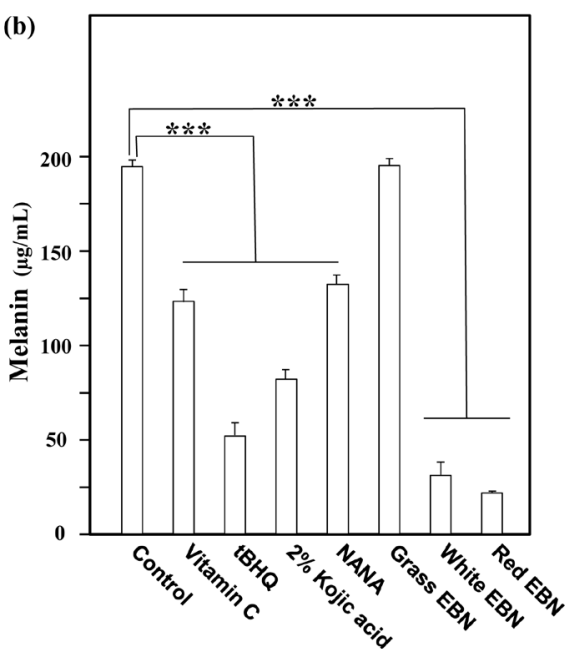

(c)
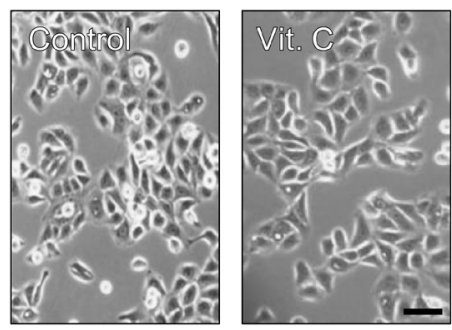

(d)

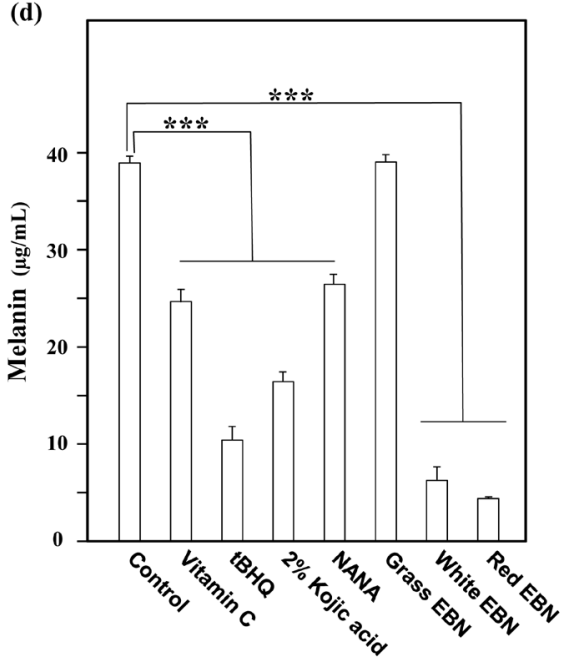

Figure 4. NANA and EBN inhibit melanin formation in cultured B16 and A375 cells. (a) B16 murine melanoma and (c) A375 human melanoma cells were seeded on 6-well plate and cultured in DMEM supplemented with $10 \%$ fetal bovine serum. On the following day, B16 cells were exposed to vitamin C (5 mM), as a positive control. For A375 human melanoma, $10 \mathrm{nM}$ of $\alpha$-MSH was applied to all samples to stimulate melanogenesis on day 2. Microscopic views of different wells were captured after 48 hours of incubation. Images were captured from intact B16 cells to observe the release of melanosomes. Scale bar $=100 \mu \mathrm{m}$. (b) Melanin from B16 cells and (d) A375 cells with or without the treatment of vitamin C (5 mM), or tBHQ $(50 \mu \mathrm{M})$, or $2 \%$ kojic acid, or NANA (20 mM), or EBN extracts $(1 \mathrm{mg} / \mathrm{mL})$, were extracted and measured by spectrometer at $405 \mathrm{~nm}$. Melanin concentrations were converted from the absorbance of $405 \mathrm{~nm}$ using melanin standard curve. The content of melanin were presented as Mean \pm SD $(n=3)$. One-way ANOVA was performed on the data set by GraphPad 5.0. Statistical significant differences were indicated. $* * * \mathrm{P}<0.001$ versus reference group.

conjugated form of NANA, and/or there are other active ingredients within EBN that perform skin lightening function synergistically with NANA.

NANA was reported to play functional roles in physiological developments in human. NANA is usually the terminal residue of cellular glycocalyx and plays important role for cellular recognition. Moreover, NANA is a major component for brain development. Disorder of NANA regulation results in retardation of brain development [18] [19]. Furthermore, NANA was reported for its functions in anti-viral [8], transformation of lymphocyte [20], growth of CaCo-2 cells [10], development of human adipose-derived stem cells [21] and proliferation of corneal keratinocytes [22]. However, no report had been found on the skincare function of NANA. In the past several years, $2 \%$ hydroquinone was accepted as a golden standard for skin lightening [23]. However, the drug was banned by FDA in 2006 because of its potential carcinogenicity. A derivative of hydroquinone with the addition of glycoside, named arbutin, was then developed into a substitute. However, the low skin lightening efficiency of arbutin had always been complained. Subsequently, the development of new skin lightening agents is an urgent need in the cosmetic market. New agents like gallic acid from gallnut and kojic acid from the by-products 
(a)

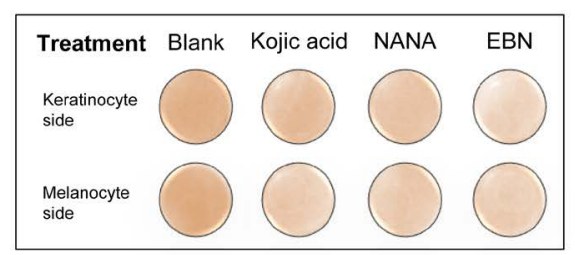

(b)
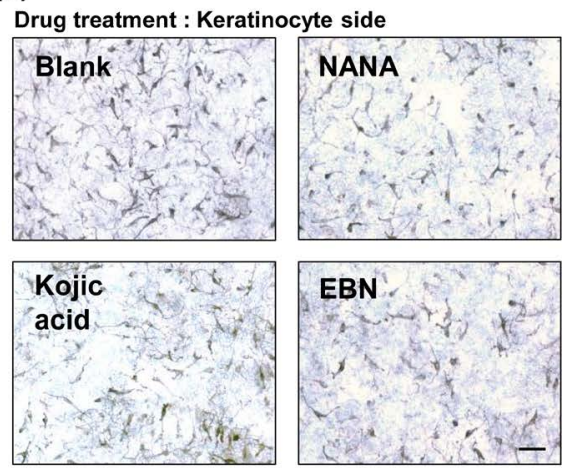

Drug treatment : Melanocyte side
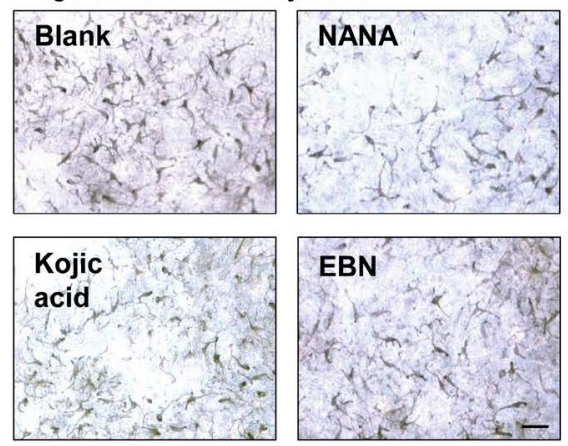

Figure 5. NANA and EBN inhibit melanin formation in 3D human skin model Blank control, $20 \mathrm{mM}$ NANA, $1 \mathrm{mg} / \mathrm{mL}$ EBN extract and $2 \%$ of kojic acid (positive control) were applied either to the inner chambers of trans-well containing the 3D human skin model (keratinocyte side) or culture medium directly (melanocyte side). Macroscopic and microscopic images were captured on day 18 after treatment. (a) Macroscopic images were captured by the top view (keratinocyte side), (b) while microscopic images were captured by inverted microscope under $100 \times$ magnification (melanocyte side). Scale bar $=$ $100 \mu \mathrm{m}, n=3$.

of Japanese rice wine (i.e. Sake) were developed for their skin lightening functions [24]. However, the mechanisms of tyrosinase inhibition of gallic acid and kojic acid is still under investigation. Here, we discovered that NANA could be a potential new skin lightening agent. Firstly, NANA is derived from natural source. Secondly, the skin lightening efficiency of NANA is comparable to known skin lightening agents. Lastly, the inhibition mechanism of NANA on tyrosinase activity is different from the inhibition mechanism of hydroquinone and arbutin (competitive inhibition). In addition, the chemical structure of NANA is different to other skin lightening agents, i.e. a phenolic ring was replaced by a glucose ring. Thus, NANA should have a high potential to be developed into a new skin lightening agent.

\section{Acknowledgements}

Supported by Hong Kong Research Grants Council Theme-based Research Scheme (T13-607/12R), ITF (UIM/ 
254), GRF (661110, 662911, 660411, 663012, 662713, M-HKUST604/13), TUYF12SC02, TUYF12SC03, TUYF15SC01, The Hong Kong Jockey Club Charities Trust (HKJCCT12SC01) and Foundation of The Awareness of Nature (TAON12SC01) to Karl Tsim. Zach Wong received a scholarship from HKJEBN Scholarship for Health and Quality Living.

\section{References}

[1] Marcone, M.F. (2005) Characterization of the Edible Bird’s Nest the "Caviar of the East”. Food Research International, 38, 1125-1134. http://dx.doi.org/10.1016/j.foodres.2005.02.008

[2] Lin, J.R., Zhou, H. and Lai, X. (2006) Application of Stereoscopy on Edible Bird's Nest Identification. Journal of Chinese Medicinal Materials, 29, 219-221.

[3] Lin, J.R., Dong, Y., Zhou, H., Lai, X.P. and Wang, P.X. (2006) Identification of Edible Bird's Nest by Electrophoresis. World Science Technology/Modernization of Traditional Chinese Medicine, 8, 30-32.

[4] Lin, J.R., Zhou, H., Lai, X.P., Hou, Y., Xian, X.M., Chen, J.N., Wang, P.X., Zhou, L. and Dong, Y. (2009) Genetic Identification of Edible Birds’ Nest Based on Mitochondrial DNA Sequences. Food Research International, 42, 10531061. http://dx.doi.org/10.1016/j.foodres.2009.04.014

[5] Chan, G.K. (2013) The Quality Assurance of Edible Bird's Nest: Removal of Nitrite Contamination and Identification of an Indicative Chemical Marker. Ph.D. Thesis, The Hong Kong University of Science and Technology, Hong Kong.

[6] Chan, G.K., Zheng, K.Y., Zhu, K.Y., Dong, T.T. and Tsim, K.W. (2013) Determination of Free N-Acetylneuraminic Acid in Edible Bird Nest: A Development of Chemical Marker for Quality Control. Journal of Ethnobiology and Traditional Medicine, 120, 620-628.

[7] Yang, M., Cheung, S.H., Li, S.C. and Cheung, H.Y. (2014) Establishment of a Holistic and Scientific Protocol for the Authentication and Quality Assurance of Edible Bird's Nest. Food Chemistry, 151, 271-278. http://dx.doi.org/10.1016/j.foodchem.2013.11.007

[8] Guo, C.T., Takahashi, T., Bukawa, W., Takahashi, N., Yagi, H., Kato, K., Hidari, K.I., Miyamoto, D., Suzuki, T. and Suzuki, Y. (2006) Edible Bird’s Nest Extract Inhibits Influenza Virus Infection. Antiviral Research, 70, 140-146. http://dx.doi.org/10.1016/j.antiviral.2006.02.005

[9] Yagi, H., Yasukawa, N., Yu, S.Y., Guo, C.T., Takahashi, N., Takahashi, T., Bukawa, W., Suzuki, T., Khoo, K.H., Suzuki, Y. and Kato, K. (2008) The Expression of Sialylated High-Antennary N-Glycans in Edible Bird's Nest. Carbohydrate Research, 343, 1373-1377. http://dx.doi.org/10.1016/j.carres.2008.03.031

[10] Rashed, A.A. and Nazaimoon, W.M. (2010) Effect of Edible Bird's Nest on Caco-2 Cell Proliferation. Journal of Food Technology, 8, 126-130. http://dx.doi.org/10.3923/jftech.2010.126.130

[11] Chan, G.K., Zhu, K.Y., Chou, D.J., Guo, A.J., Lau, D.T., Dong, T.T. and Tsim, K.W. (2013) Surveillance of Nitrite Level on Edible Bird's Nest in Hong Kong: Evaluation of Removal Method and Proposed Origin of Contamination. Food Control, 34, 637-644. http://dx.doi.org/10.1016/j.foodcont.2013.06.010

[12] Chen, Q.X., Song, K.K., Qiu, L., Liu, X.D., Huang, H. and Guo, H.Y. (2005) Inhibitory Effects on Mushroom Tyrosinase by p-Alkoxybenzoic acids. Food Chemistry, 91, 269-274. http://dx.doi.org/10.1016/j.foodchem.2004.01.078

[13] Iwata, M., Corn, T., Iwata, S., Everett, M.A. and Fuller, B.B. (1990) The Relationship between Tyrosinase Activity and Skin Color in Human Foreskins. Journal of Investigative Dermatology, 85, 9-15. http://dx.doi.org/10.1111/1523-1747.ep12872677

[14] Jeon, S.H., Kim, K.H., Koh, J.U. and Kong, K.H. (2005) Inhibitory Effects on L-Dopa Oxidation of Tyrosinase by Skin-Whitening Agents. Bulletin of the Korean Chemical Society, 26, 1135-1137. http://dx.doi.org/10.5012/bkcs.2005.26.7.1135

[15] Moon, J.Y., Yim, E.Y., Song, G., Lee, N.H. and Hyun, C.G. (2010) Screening of Elastase and Tyrosinase Inhibitory Activity from Jeju Island Plants. EurAsian Journal of Biological Sciences, 4, 41-53. http://dx.doi.org/10.5053/ejobios.2010.4.0.6

[16] Kasraee, B., Hügin, A., Tran, C., Sorg, O. and Saura, J.H. (2004) Methimazole Is an Inhibitor of Melanin Synthesis in Cultured B16 Melanocytes. Journal of Investigative Dermatology, 122, 1338-1341. http://dx.doi.org/10.1111/j.0022-202X.2004.22509.x

[17] Leung, C.Y. (2004) Three Billions Market Competition for Edible Bird's Nest Shops. Economic Digest, 1197, 68-69.

[18] Ham, M., Prinsen, B.H., Huijmans, J.G., Abeling, N.G., Dorland, B., Berger, R., Koning, T.J. and Sain-van Der Velden, M.G. (2007) Quantification of Free and Total Sialic Acid Excretion by LC-MS/MS. Journal of Chromatography B, 848, 251-257. http://dx.doi.org/10.1016/j.jchromb.2006.10.066

[19] Sillanaukee, P., Pönniö, M. and Jääskeläinen, I.P. (1999) Occurrence of Sialic Acids in Healthy Humans and Different Disorders. European Journal of Clinical Investigation, 29, 413-425. 
http://dx.doi.org/10.1046/j.1365-2362.1999.00485.x

[20] Hou, Y., Xian, X., Lin, J., Lai, X. and Chen, J. (2010) The Effects of Edible Birds' Nest (Aerodramus) on Con A-Induced Rats’ Lymphocytes Transformation. China Modern Medicine, 17, 9-11.

[21] Roh, K.B., Lee, J., Kim, Y.S., Park, J., Kim, J.H., Lee, J. and Park, D. (2011) Mechanisms of Edible Bird’s Nest Extract-Induced Proliferation of Human Adipose-Derived Stem Cells. Evidence-Based Complementary Alternative Medicine, 2012, Article ID: 797520. http://dx.doi.org/10.1155/2012/797520

[22] Fadhilah, Z.A., Chua, K.H., Ng, S.L., Elvy, S.M., Lee, T.H. and Norzana, A.G. (2011) Effects of Edible Bird's Nest (EBN) on Cultured Rabbit Corneal Keratinocytes. BMC Complementary and Alternative Medicine, 11, 1-11.

[23] Makino, E.T., Mehta, R.C., Banga, A., Jain, P., Sigler, M.L. and Sonti, S. (2013) Evaluation of a Hydroquinone-Free Skin Brightening Product Using in Vitro Inhibition of Melanogenesis and Clinical Reduction of Ultraviolet-Induced Hyperpigmentation. Journal of Drugs in Dermatology, 12, s16-s20.

[24] Kumar, K.J., Vani, M.G., Wang, S.Y., Liao, J.W., Hsu, L.S., Yang, H.L. and Hseu, Y.C. (2013) In Vitro and in Vivo Studies Disclosed the Depigmenting Effects of Gallic Acid: A Novel Skin Lightening Agent for Hyperpigmentary Skin Diseases. BioFactors, 39, 259-270. http://dx.doi.org/10.1002/biof.1064 


\section{Supplementary}
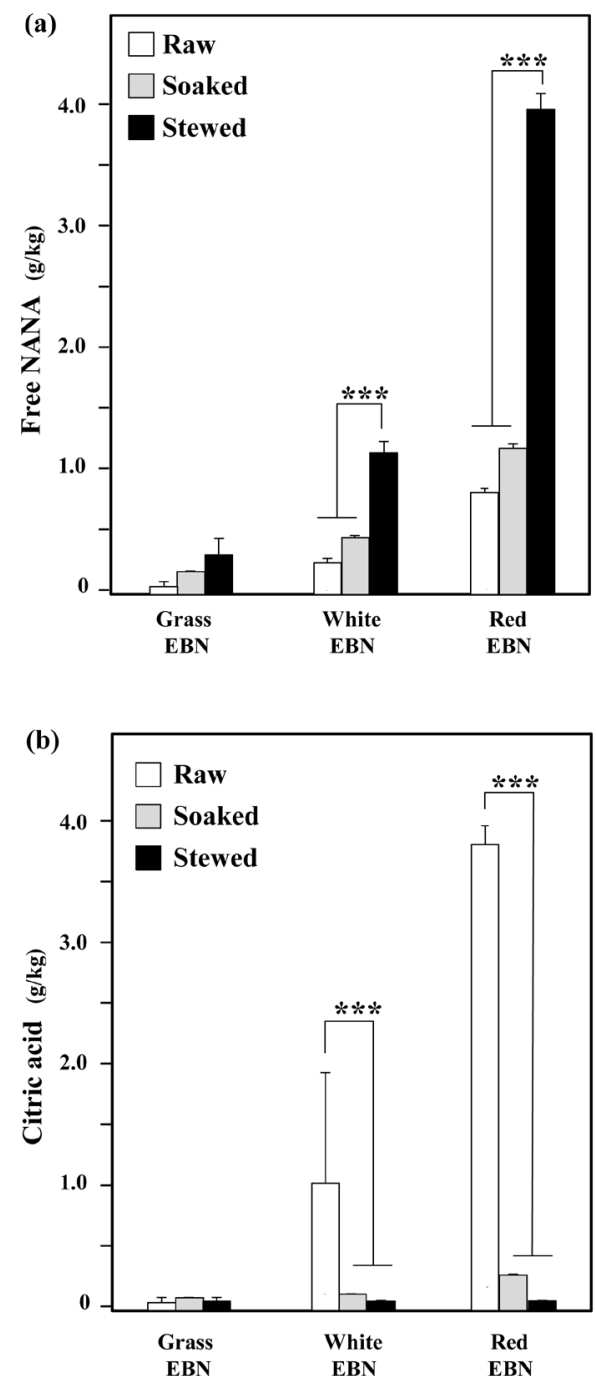

Supplementary Figure 1. The contents of NANA and citric acid during cooking. Twelve batches purchased EBN were processed by standard procedure. For Grass and White EBN, EBNs were soaked in 100 folds of water (w/v) for 3 hours and stewed in 30 folds of water for 0.5 hour. For Red EBN, EBN were soaked for 15 hours and stewed in 30 folds of water for 2.5 hours. The content of free NANA (A) and citric acid (B) on Grass EBN, White EBN and Red EBN during different stages of processing were determined. The free NANA and citric acidin $\mathrm{g} / \mathrm{kg}$ of dry material was presented as Mean + SD (n $=3)$. $* * * \mathrm{P}<0.001$ versus reference group.

\section{Supplementary Table. The contents of protein, free NANA and citric acid of different grades of EBN.}

\begin{tabular}{|c|c|c|c|c|c|c|}
\hline \multirow[t]{2}{*}{ EBN Type $^{\mathrm{a}}$} & \multicolumn{2}{|c|}{ Protein $^{\mathrm{b}}$ (g/kg) } & \multicolumn{2}{|c|}{ Free NANA $(\mathrm{mg} / \mathrm{kg})$} & \multicolumn{2}{|c|}{ Citric acid $(\mathrm{g} / \mathrm{kg})$} \\
\hline & Range & Medium & Range & Medium & Range & Medium \\
\hline Grass EBN & $314.52-558.81$ & 507.11 & $51.35-130.03$ & 54.31 & 0.00 & 0.00 \\
\hline White EBN & $507.28-695.04$ & 680.53 & $96.57-691.23$ & 170.46 & $0.00-2.04$ & 1.00 \\
\hline Red EBN & $459.27-504.00$ & 477.32 & $310.26-910.81$ & 802.51 & $1.05-4.03$ & 3.24 \\
\hline
\end{tabular}

${ }^{\mathrm{a}}$ Twelve batches of EBN were randomly purchased from local market of Hong Kong, at least three batches for each type of EBN. The original production countries included Indonesia, Malaysia, Vietnam, Thailand and Philippines. 'Protein content of EBN was measured by Bradford's method after completely solubilized by stewing. ${ }^{c}$ Free NANA content was measured by calculating the peak area in LC-MS/MS chromatogram with free NANA standard. ${ }^{\mathrm{d}}$ Citric acid content was measured by calculating the peak area in LC-MS/MS chromatogram with citric acid standard. All contents were presented as per kilogram of crude EBN, the contents of each items were presented $(n=3)$. 